\title{
Glioblastoma in the elderly: the effect of aggressive and modern therapies on survival
}

\author{
Ranjith Babu, MD, MS, ${ }^{1}$ Jordan M. Komisarow, MD, ${ }^{1}$ Vijay J. Agarwal, MD, ${ }^{1}$ \\ Shervin Rahimpour, MD, ${ }^{1}$ Akshita lyer, BS, ${ }^{1}$ Dylan Britt, BS, ${ }^{1}$ Isaac O. Karikari, MD, ${ }^{1}$ \\ Peter M. Grossi, MD, ${ }^{1}$ Steven Thomas, MS, ${ }^{2}$ Allan H. Friedman, MD, ${ }^{1}$ and \\ Cory Adamson, MD, PhD, MPH, MHSc ${ }^{1,3,4}$
}

1Division of Neurosurgery, Department of Surgery, and 2Department of Biostatistics and Bioinformatics, DUMC, Duke University School of Medicine, Durham, North Carolina; ${ }^{3}$ Neurosurgery, Atlanta VA Medical Center, Decatur; and ${ }^{4}$ Department of Neurosurgery, Emory University, Atlanta, Georgia

OBJECTIVE The prognosis of elderly patients with glioblastoma (GBM) is universally poor. Currently, few studies have examined postoperative outcomes and the effects of various modern therapies such as bevacizumab on survival in this patient population. In this study, the authors evaluated the effects of various factors on overall survival in a cohort of elderly patients with newly diagnosed GBM.

METHODS A retrospective review was performed of elderly patients ( $\geq 65$ years old) with newly diagnosed GBM treated between 2004 and 2010. Various characteristics were evaluated in univariate and multivariate stepwise models to examine their effects on complication risk and overall survival.

RESULTS A total of 120 patients were included in the study. The median age was 71 years, and sex was distributed evenly. Patients had a median Karnofsky Performance Scale (KPS) score of 80 and a median of 2 neurological symptoms on presentation. The majority $(53.3 \%)$ of the patients did not have any comorbidities. Tumors most frequently (43.3\%) involved the temporal lobe, followed by the parietal (35.8\%), frontal (32.5\%), and occipital (15.8\%) regions. The majority (57.5\%) of the tumors involved eloquent structures. The median tumor size was $4.3 \mathrm{~cm}$. Every patient underwent resection, and $63.3 \%$ underwent gross-total resection (GTR). The vast majority (97.3\%) of the patients received the postoperative standard of care consisting of radiotherapy with concurrent temozolomide. The majority $(59.3 \%)$ of patients received additional agents, most commonly consisting of bevacizumab (38.9\%). The median survival for all patients was 12.0 months; $26.7 \%$ of patients experienced long-term ( $\geq 2$-year) survival. The extent of resection was seen to significantly affect overall survival; patients who underwent GTR had a median survival of 14.1 months, whereas those who underwent subtotal resection had a survival of 9.6 months $(p=0.038)$. Examination of chemotherapeutic effects revealed that the use of bevacizumab compared with no bevacizumab $(20.1$ vs 7.9 months, respectively; $p<0.0001)$ and irinotecan compared with no irinotecan (18.0 vs 9.7 months, respectively; $p=0.027$ ) significantly improved survival. Multivariate stepwise analysis revealed that older age (hazard ratio [HR] 1.06 [95\% $\mathrm{Cl} 1.02-1.10] ; p=0.0077$ ), a higher KPS score (HR 0.97 [95\% $\mathrm{Cl} 0.95-0.99] ; p=0.0082$ ), and the use of bevacizumab ( $\mathrm{HR} 0.51$ [95\% $\mathrm{Cl} 0.31-0.83] ; p=0.0067$ ) to be significantly associated with survival.

CONCLUSION This study has demonstrated that GTR confers a modest survival benefit on elderly patients with GBM, suggesting that safe maximal resection is warranted. In addition, bevacizumab significantly increased the overall survival of these elderly patients with GBM; older age and preoperative KPS score also were significant prognostic factors. Although elderly patients with GBM have a poor prognosis, they may experience enhanced survival after the administration of the standard of care and the use of additional chemotherapeutics such as bevacizumab.

http://thejns.org/doi/abs/10.3171/2015.4.JNS142200

KEY WORDS bevacizumab; elderly; glioblastoma; oncology; prognostic factors

ABBREVIATIONS EOR = extent of resection; GBM = glioblastoma; GTR = gross-total resection; HR = hazard ratio; KPS = Karnofsky Performance Scale; RT = radiotherapy; SRS = stereotactic radiosurgery; STR = subtotal resection; TMZ = temozolomide.

SUBMITTED October 12, 2014. ACCEPTED April 8, 2015.

INCLUDE WHEN CITING Published online October 9, 2015; DOI: 10.3171/2015.4.JNS142200. 
$\mathrm{G}$ LIOBLASTOMA (GBM) is the most common primary brain tumor in adults and most commonly occurs in individuals aged 65 years or older. ${ }^{50}$ Despite maximal therapy consisting of resection and aggressive radiochemotherapy, the median survival remains approximately 14 months. ${ }^{1}$ Although the prognosis of GBM is universally poor, elderly patients have been shown to have significantly worse outcomes than younger patients; their median survival ranges from 4 to 9 months. ${ }^{4,20,21,40,46}$ As the incidence of GBM has been rising in elderly patients while this population group rapidly expands, it is increasingly important to identify prognostic factors and effective therapeutic strategies for improving survival in elderly patients. ${ }^{5,18}$

Current therapy for GBM consists of maximal safe resection followed by radiotherapy (RT) and concomitant temozolomide (TMZ). However, the optimum treatment strategy for elderly patients is unclear because of the available data, which are limited for several reasons. First, most clinical trials have excluded elderly patients; the landmark European Organization for Research and Treatment of Cancer (EORTC) 26981-22981/National Cancer Institute of Canada Clinical Trials Group (NCIC) CE3 randomized Phase III trial excluded patients aged 70 years or older. ${ }^{44}$ Second, elderly patients are less likely to receive standard therapy and multiagent treatment because of the fear of treatment toxicity. ${ }^{2,21}$ Finally, many elderly patients are offered only stereotactic biopsy or conservative surgical treatment out of concern for increased complications and their inability to tolerate extensive procedures..$^{20,34,40,46}$

Although few studies have demonstrated that an increasing extent of resection (EOR) improves survival in elderly patients, no studies have investigated the effects of modern therapies on the survival of elderly patients with GBM. In particular, the effect of bevacizumab in this patient population is of interest because elderly patients have more angiogenic tumors than those who are younger. In this study, we evaluated the effects of various patient, tumor, and treatment factors on overall survival in a cohort of elderly patients with newly diagnosed GBM.

\section{Methods \\ Patient Cohort}

This study was approved by the Duke University School of Medicine Institutional Review Board. Elderly patients (aged 65 years or older) with newly diagnosed primary GBM who underwent primary resection at the Duke University Medical Center between 2004 and 2010 were identified. Any patient who underwent only stereotactic biopsy, had a previous resection and/or adjuvant therapy, or had been diagnosed previously with a lower-grade glioma was excluded. A total of 120 patients met the inclusion criteria and were the subjects of an institutional review board-approved chart review.

\section{Recorded Variables}

The medical records of each patient were reviewed, and various patient, tumor, and treatment variables were recorded. Tumor location was determined by using preoperative MRI. Tumors that significantly involved the sensorimotor strip (precentral and postcentral gyri), perisylvian language areas (including the inferior frontal, superior temporal, and inferior parietal areas in the dominant hemisphere), the thalamus, or the calcarine visual cortex were considered to have an eloquent location. Resection in these areas involved intraoperative cortical mapping when applicable. Tumor size was determined by the largest measured diameter in any plane on contrast-enhanced MR images. Preoperative symptoms recorded included headache, ataxia/gait disturbance, new-onset seizures, progressive cognitive difficulty, and motor, sensory, or language deficits. The preoperative Karnofsky Performance Scale (KPS) score was also used to categorize patient functional status. Recorded patient comorbidities included congestive heart failure, previous myocardial infarction, peripheral vascular disease, cerebrovascular disease (including cerebrovascular accidents and transient ischemic attacks), dementia, chronic obstructive pulmonary disease (including asthma, emphysema, and chronic bronchitis), connective tissue disease, peptic ulcer disease, mild and moderate-to-severe liver disease, diabetes with or without end-organ damage, hemiplegia, moderate-to-severe renal disease, history of previous localized tumors within 5 years of GBM diagnosis, leukemia, lymphoma, metastatic cancer, and AIDS. These comorbidities were then used to calculate the Charlson Comorbidity Index, higher scores of which are associated with higher mortality risk. ${ }^{6}$ The EOR was classified as gross-total resection (GTR) (> 99\% contrast enhancement) or subtotal resection (STR) by evaluating preoperative and postoperative T1-weighted Gd-enhanced MRI studies. The most common chemotherapeutics used at any time after surgery in combination with or following the standard of care were recorded. The number of other chemotherapeutics used was defined as the number of non-TMZ chemotherapies administered. Other treatments recorded included the use of carmustine wafers placed in the resection cavity and stereotactic radiosurgery (SRS). Overall survival was defined as the time from histopathological diagnosis to death or the censored date of the last follow-up.

\section{Statistical Analysis}

The data were summarized using medians for continuous variables and counts and percentages for categorical variables. Differences in continuous variables were analyzed by using the Mann-Whitney U test, and categorical variables were evaluated by using the chi-square test.

Survival was estimated by using the Kaplan-Meier method, and the log-rank test was used to evaluate the differences between survival curves. Stepwise multivariate Cox proportional hazard models were used to calculate hazard ratios (HRs) and 95\% confidence intervals (CIs) to investigate independent predictors of overall survival. A p value of $<0.1$ was required for variable entry and retention in all stepwise multivariate models. Any $\mathrm{p}$ value of $<0.05$ was considered statistically significant. All analyses were performed by using SAS 9.3 (SAS Institute).

\section{Results \\ Patient Cohort}

A total of 120 patients met inclusion criteria, and their 
TABLE 1. Cohort characteristics of elderly patients ( $\geq 65$ years old) with $\mathrm{GBM}^{*}$

\begin{tabular}{|c|c|}
\hline Characteristic & Data \\
\hline \multicolumn{2}{|l|}{ Demographics } \\
\hline Age (yrs) & 71 \\
\hline Female & $49.2(59)$ \\
\hline \multicolumn{2}{|l|}{ Presentation } \\
\hline KPS score & $80(45-100)$ \\
\hline Neurological symptoms & 2 \\
\hline Weakness & $19.2(23)$ \\
\hline Headache & $25.8(31)$ \\
\hline Ataxia/gait instability & $25.8(31)$ \\
\hline Sensory disturbance & $23.3(28)$ \\
\hline Sensorimotor & $2.5(3)$ \\
\hline Language & $30.8(37)$ \\
\hline Seizures & $20.8(25)$ \\
\hline Cognition & $45.8(55)$ \\
\hline \multicolumn{2}{|l|}{ Comorbidities } \\
\hline \multicolumn{2}{|l|}{ Charlson score } \\
\hline 0 & $53.3(64)$ \\
\hline 1 & $25.0(30)$ \\
\hline 2 & $15.0(18)$ \\
\hline$\geq 3$ & $6.7(8)$ \\
\hline Congestive heart failure & $0.8(1)$ \\
\hline Myocardial infarction & $4.2(5)$ \\
\hline Peripheral vascular disease & $6.7(8)$ \\
\hline Cerebrovascular disease & $3.3(4)$ \\
\hline Dementia & $0.8(1)$ \\
\hline COPD & $8.3(10)$ \\
\hline Connective tissue disease & $1.7(2)$ \\
\hline Peptic ulcer disease & $3.3(4)$ \\
\hline Mild liver disease & $0.0(0)$ \\
\hline Diabetes, uncomplicated & $20.8(25)$ \\
\hline Hemiplegia & $0.0(0)$ \\
\hline Diabetes, end-organ damage & $0.8(1)$ \\
\hline Renal disease & $2.5(3)$ \\
\hline Tumor without metastasis & $10.0(12)$ \\
\hline Leukemia & $0.0(0)$ \\
\hline Lymphoma & $0.0(0)$ \\
\hline Moderate-to-severe liver disease & $0.0(0)$ \\
\hline Metastatic tumor & $0.0(0)$ \\
\hline AIDS & $0.0(0)$ \\
\hline \multicolumn{2}{|l|}{ Tumor } \\
\hline \multicolumn{2}{|l|}{ Involved locations } \\
\hline Frontal & $32.5(39)$ \\
\hline Temporal & $43.3(52)$ \\
\hline Parietal & $35.8(43)$ \\
\hline Occipital & $15.8(19)$ \\
\hline Corpus callosum & $16.7(20)$ \\
\hline Eloquent & $57.5(69)$ \\
\hline
\end{tabular}

TABLE 1. Cohort characteristics of elderly patients ( $\geq 65$ years old) with GBM* (continued)

\begin{tabular}{|c|c|}
\hline Characteristic & Data \\
\hline \multicolumn{2}{|l|}{ Tumor (continued) } \\
\hline Multilobar & $24.2(29)$ \\
\hline Size (cm) & 4.3 \\
\hline \multicolumn{2}{|l|}{ Surgical treatment } \\
\hline Resection & $100(120)$ \\
\hline \multicolumn{2}{|l|}{ EOR } \\
\hline GTR & $63.3(76)$ \\
\hline STR & $36.7(44)$ \\
\hline Re-resection & $2.5(3)$ \\
\hline \multicolumn{2}{|l|}{ Adjuvant treatment $\dagger$} \\
\hline RT & $98.2(111)$ \\
\hline TMZ & $98.2(111)$ \\
\hline $\mathrm{RT} \& \mathrm{TMZ}$ & $97.3(110)$ \\
\hline Bevacizumab & $38.9(44)$ \\
\hline Initial/before progression & $19.5(22)$ \\
\hline After progression & $19.5(22)$ \\
\hline Lomustine & $10.6(12)$ \\
\hline After progression & $10.6(12)$ \\
\hline Irinotecan & $21.2(24)$ \\
\hline Initial/before progression & $7.1(8)$ \\
\hline After progression & $14.2(16)$ \\
\hline Etoposide & $18.6(21)$ \\
\hline After progression & $18.6(21)$ \\
\hline Immunotherapy & $5.3(6)$ \\
\hline Before progression & $5.3(6)$ \\
\hline Other chemotherapy & $59.3(67)$ \\
\hline Carmustine wafer & $0.88(1)$ \\
\hline SRS & $7.1(8)$ \\
\hline Chemotherapeutics (mean) & 1.4 \\
\hline
\end{tabular}

COPD = chronic obstructive pulmonary disease.

* The total number of patients was 120. Data shown are median (range) or percentage (number) unless stated otherwise.

$\dagger$ The total number of patients was 113 .

data were analyzed. The median age was 71 years (range 65-89 years), and sex was distributed evenly (49.2\% female) (Table 1). The patients had a median KPS score of 80 and a median of 2 neurological symptoms at presentation. The most common presenting symptoms were cognitive difficulties (45.8\%), language dysfunction (30.8\%), headache (25.8\%), and ataxia/gait instability (25.8\%). The majority (53.3\%) of the patients did not have a comorbidity, and the most common comorbidities were uncomplicated diabetes (20.8\%), history of nonmetastatic tumors (10.0\%), and chronic obstructive pulmonary disease $(8.3 \%)$. Tumors most frequently (43.3\%) involved the temporal lobe, followed by the parietal (35.8\%), frontal (32.5\%), and occipital $(15.8 \%)$ regions. The majority $(57.5 \%)$ of the tumors involved eloquent structures, whereas $16.7 \%$ of them significantly affected the corpus callosum. The median tumor size was $4.3 \mathrm{~cm}$. 
TABLE 2. Outcome characteristics of elderly patients ( $\geq 65$ years old) with $\mathrm{GBM}^{*}$

\begin{tabular}{cl}
\hline Characteristic & Value \\
\hline Recurrence/progression & $59.2(71)$ \\
\hline Survival (mos) & 12.0 \\
\hline 1 yr & $50.0(60)$ \\
\hline 2 yrs & $26.7(32)$ \\
\hline 3 yrs & $10.0(12)$ \\
\hline
\end{tabular}

* The total number of patients was 120. Data shown are median number of months or percentage (number) of patients.

Each patient underwent resection; $63.3 \%$ underwent GTR. The vast majority (97.3\%) of the patients received the postoperative standard of care consisting of RT with concurrent TMZ (range $75-300 \mathrm{mg} / \mathrm{m}^{2}$ ). The majority $(59.3 \%)$ of the patients received additional agents, most commonly consisting of bevacizumab (38.9\%; range $10-15 \mathrm{mg} / \mathrm{kg}$ ), irinotecan (21.2\%; range $\left.70-125 \mathrm{mg} / \mathrm{m}^{2}\right)$, etoposide $\left(18.6 \% ; 50 \mathrm{mg} / \mathrm{m}^{2}\right)$, lomustine $(10.6 \% ; 110 \mathrm{mg}$ / $\mathrm{m}^{2}$ ), and immunotherapy (5.3\%). These chemotherapeutics were administered most frequently after progression (65.7\%); the remaining patients received 1 or more of these agents initially with TMZ. After progression, 2.5\% of the patients underwent re-resection; 1 of these patients received carmustine wafers in the resection cavity. Some patients $(7.1 \%)$ also underwent SRS after focal tumor progression.

\section{Survival}

The median survival for all patients was 12.0 months; $26.7 \%$ of the patients experienced long-term ( $\geq 2$-year) survival (Table 2; Fig. 1). Univariate analysis revealed that a variety of patient and treatment factors significantly affected survival (Table 3). Patients 75 years of age or older had significantly worse survival than younger patients (7.9 vs 15.1 months, respectively; $p<0.0001$, and women experienced longer survival than men (15.7 vs 10.5 months, respectively; $\mathrm{p}=0.025$ ) (Fig. 2 left). Those presenting with a higher KPS score had increased survival times; 21.8 months for a KPS score of $\geq 90,12.2$ months for a KPS score of 80, 7.2 months for a KPS score of 70, and 7.4 months for a KPS score of $<70$ (Fig. 2 right). The EOR was also found to significantly affect overall survival; patients who underwent GTR had a median survival of 14.1 months, whereas those who underwent STR had a survival of 9.6 months $(p=0.038)$ (Fig. 3 left). An examination of chemotherapeutic effects revealed that the use of bevacizumab compared with no bevacizumab (20.1 vs 7.9 months, respectively; $\mathrm{p}<0.0001$ ) and irinotecan compared with no irinotecan (18.0 vs 9.7 months, respectively; $p=0.027$ ) significantly improved survival (Fig. 4A and B). Although etoposide compared with no etoposide (20.9 vs 9.9 months, respectively; $\mathrm{p}=0.052$ ) and immunotherapy compared with no immunotherapy $(24.8$ vs 10.6 months, respectively; $\mathrm{p}=0.17$ ) extended survival, these effects were not significant (Fig. 4C and D). The use of chemotherapeutic agents following the standard of care

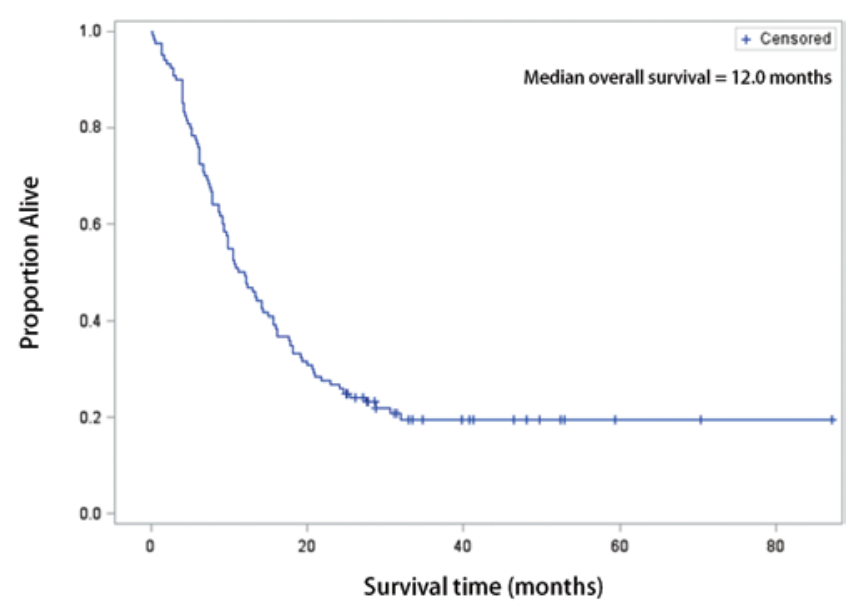

FIG. 1. Kaplan-Meier overall survival curve for all elderly patients in the study with a newly diagnosed GBM. Figure is available in color online only.

significantly improved survival over those who received no chemotherapeutic agents (17.7 vs 7.2 months, respectively; $\mathrm{p}=0.0004$ ), and the use of more than 2 chemotherapeutics compared with those who received 2 or fewer chemotherapeutics provided a significant survival benefit (21.3 vs 9.4 months, respectively; $\mathrm{p}=0.018$ ) (Fig. 4E and F). The administration of SRS after focal tumor progression also improved overall survival over those who did not undergo SRS (median not reached vs 10.5 months, respectively; $\mathrm{p}=0.0006)$ (Fig. 3 right).

Multivariate stepwise analysis was used to identify independent predictors of survival; GTR was forced into the model because of its prognostic significance (Table 4). Consistent with the univariate analysis, older age was associated with significantly worse survival (HR 1.06 [95\% CI 1.02-1.10]; $\mathrm{p}=0.0077$ ), whereas a higher preoperative KPS score was a significant favorable prognostic factor (HR 0.97 [95\% CI 0.95-0.99]; $p=0.0082$ ). In addition, the use of bevacizumab was independently associated with significantly improved survival (HR 0.51 [95\% CI 0.310.83 ]; $\mathrm{p}=0.0067)$. Although GTR also improved survival, its effect was not statistically significant (HR 0.70 [95\% CI $0.46-1.08] ; \mathrm{p}=0.10$ ).

\section{Discussion}

The treatment of elderly patients has yet to be clearly defined because of the lack of treatment guidelines, which may be a result of the frequent exclusion of this patient population from large randomized trials. ${ }^{44}$ As a result, treatments are typically generalized to this group. However, because the incidence of GBM in elderly patients is increasing while this population group expands, it has become increasingly important to identify effective therapeutic strategies that may extend survival in this vulnerable patient population..$^{5,18}$

Older patients are often treated more conservatively than those who are younger; studies have demonstrated that these patients are significantly more likely to undergo biopsy alone while having decreased odds of receiving RT and/or chemotherapy after surgery.,21 This less aggressive 
TABLE 3. Univariate analysis of overall survival in elderly patients ( $\geq 65$ years old) with GBM

\begin{tabular}{|c|c|c|}
\hline Factor & $\begin{array}{l}\text { Median Survival } \\
\text { Time (mos) }\end{array}$ & $p$ Value \\
\hline Age at diagnosis & & $<0.0001$ \\
\hline$<75 \mathrm{yrs}$ & 15.1 & \\
\hline$\geq 75$ yrs & 7.9 & \\
\hline Sex & & 0.025 \\
\hline Male & 10.5 & \\
\hline Female & 15.7 & \\
\hline KPS score at diagnosis & & 0.0082 \\
\hline$\geq 90$ & 21.8 & \\
\hline 80 & 12.2 & \\
\hline 70 & 7.2 & \\
\hline$<70$ & 7.4 & \\
\hline Charlson Index & & 0.95 \\
\hline 0 & 10.2 & \\
\hline$\geq 1$ & 13.4 & \\
\hline Location & & 0.96 \\
\hline Frontal & 10.5 & \\
\hline Temporal & 12.2 & \\
\hline Parietal & 14.2 & \\
\hline Occipital & 17.7 & \\
\hline Eloquent & 12.1 & 0.60 \\
\hline Noneloquent & 10.7 & \\
\hline Multilobar & 14.4 & 0.93 \\
\hline Single lobe & 10.6 & \\
\hline Corpus callosum & 7.2 & 0.14 \\
\hline No involvement & 13.4 & \\
\hline Tumor size & & 0.56 \\
\hline$\leq 4.3 \mathrm{~cm}$ & 14.2 & \\
\hline$>4.3 \mathrm{~cm}$ & 10.5 & \\
\hline EOR & & 0.038 \\
\hline GTR & 14.1 & \\
\hline STR & 9.6 & \\
\hline \multicolumn{3}{|l|}{ Postoperative therapy } \\
\hline Bevacizumab & 20.1 & $<0.0001$ \\
\hline No bevacizumab & 7.9 & \\
\hline Lomustine & 14.6 & 0.43 \\
\hline No lomustine & 10.7 & \\
\hline Irinotecan & 18.0 & 0.027 \\
\hline No irinotecan & 9.7 & \\
\hline Etoposide & 20.9 & 0.052 \\
\hline No etoposide & 9.9 & \\
\hline SRS & NR & 0.0006 \\
\hline No radiosurgery & 10.5 & \\
\hline Immunotherapy & 24.8 & 0.17 \\
\hline No immunotherapy & 10.6 & \\
\hline Re-resection & NR & 0.10 \\
\hline No re-resection & 11.0 & \\
\hline Chemotherapy after SOC & 17.7 & 0.0004 \\
\hline
\end{tabular}

TABLE 3. Univariate analysis of overall survival in elderly patients ( $\geq 65$ years old) with GBM

\begin{tabular}{ccc}
\hline Factor & $\begin{array}{c}\text { Median Survival } \\
\text { Time (mos) }\end{array}$ & p Value \\
\hline Postoperative therapy (continued) & & \\
\hline No additional chemotherapy & 7.2 & \\
\hline No. of other chemotherapies & & \\
\hline$\leq 2$ & 9.4 & \\
\hline$>2$ & 21.3 & \\
\hline
\end{tabular}

$\mathrm{NR}=$ not reached; $\mathrm{SOC}=$ standard of care .

treatment may be a result of reluctance to offer aggressive surgical and medical treatment for fear of postoperative complications and drug toxicity. In addition to their reduced functional reserve and increased comorbidities, elderly patients also historically have significantly worse survival, which leads many to question the utility of aggressive interventions.

Currently, resection is the first-line treatment for GBM; the Joint Tumor Section of the American Association of Neurological Surgeons (AANS) and the Congress of Neurological Surgeons (CNS) recommend maximal safe resection with Type II evidence..$^{32}$ Both retrospective and prospective studies have consistently demonstrated more extensive resection to significantly improve outcomes. ${ }^{25-27,33,37,38,42,43}$ In a retrospective post hoc analysis of data from a prospectively randomized controlled Phase III trial, Stummer et al. ${ }^{43}$ reported that patients who underwent complete resection had significantly longer survival than those with incomplete resection (16.9 vs 11.8 months, respectively; $p<0.0001)$. Multivariate analysis also demonstrated postoperative residual tumor to be a significant poor prognostic factor (HR 1.75 [95\% CI 1.26-2.44]; $\mathrm{p}=$ $0.0009)$. More recently, the authors of a large retrospective cohort study of 500 patients reported a significant survival benefit for those with an EOR as low as $78 \% .{ }^{38}$ Stepwise improvement in survival was also noted in the 95\%-100\% EOR range. Multivariate analysis demonstrated postoperative tumor volume to be a significant predictor of survival. However, the effect of aggressive resection on the survival of elderly patients with GBM is unclear because of the very few studies that have investigated it. In a study of 361 patients with GBM (146 of whom were aged 65 years or older), elderly patients who underwent complete resection had significantly longer survival than patients who had only partial resection ( $>5 \%$ residual) (17.7 vs 11.4 months, respectively; $p=0.006) .{ }^{34}$ Complete resection was also a significant prognostic factor in multivariate analysis, whereas the effects of STR and partial resection were not significant. Similarly, others have reported that increasing the EOR provides greater benefits to survival for elderly patients with GBM..$^{20,29}$ However, although other studies demonstrated a significant survival benefit compared with biopsy only, they did not observe significant differences between GTR and STR..$^{3,10,12}$ In this study, we have demonstrated that elderly patients who received GTR had significantly longer survival than those who underwent STR only (14.1 vs 9.6 months, respectively; $\mathrm{p}=$ 

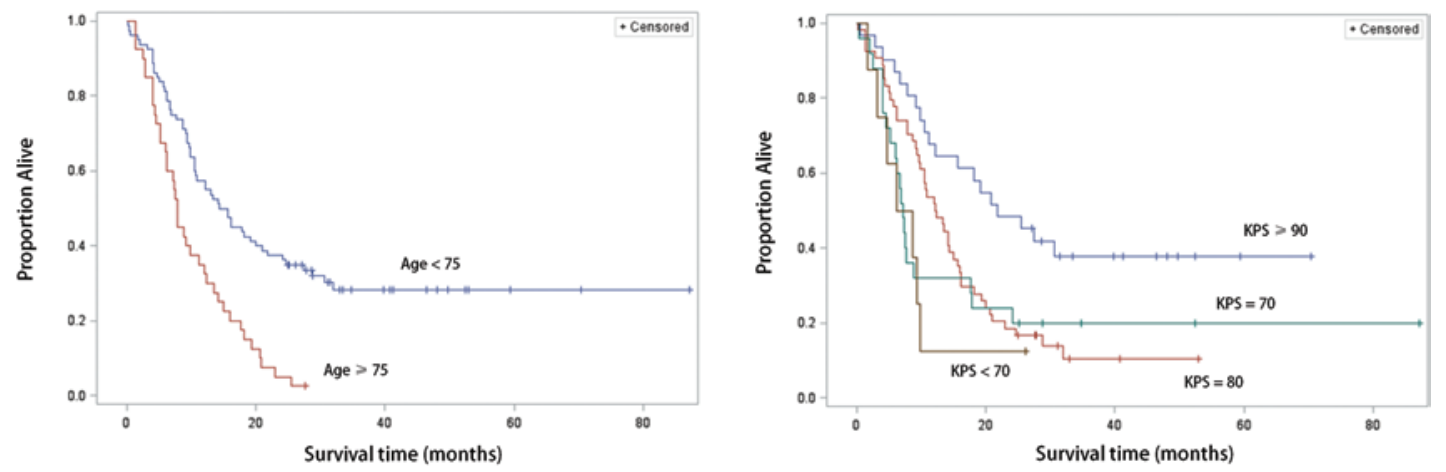

FIG. 2. Kaplan-Meier overall survival curves demonstrating significantly worse survival for patients aged 75 or older (left) and significantly worse survival for those with a lower KPS score (right). Figure is available in color online only.

0.038). Although GTR was a significant prognostic factor on multivariate analysis when controlling only for patient and tumor characteristics (data not shown), the inclusion of chemotherapeutics into the model resulted in this effect becoming nonsignificant (HR 0.70 [95\% CI 0.46-1.08]; p $=0.10$ ).

After pathological confirmation via resection or biopsy, patients with GBM typically receive RT and concurrent TMZ, the current standard of care. However, as previously stated, elderly patients commonly do not receive both RT and chemotherapy. Radiotherapy has been consistently shown to improve survival in elderly patients with GBM over that in patients undergoing supportive care alone; a randomized trial demonstrated that RT (50 Gy in 1.8-Gy fractions) provided a survival benefit without affecting cognition or quality of life. ${ }^{22}$ Because of the potential for neurotoxicity with cranial RT in elderly patients, the use of TMZ alone has also been investigated. ${ }^{8,15,17}$ A retrospective study by Glantz et al. ${ }^{17}$ examined the outcomes of 32 elderly patients who received TMZ (5-day schedule) and 54 patients who received RT only (60 Gy in 1.8-Gy fractions). The median survival times were similar between the groups; those who received TMZ only had slightly longer survival (6 vs 4.1 months, respectively; $p=0.20$ ). More recently, a Phase II trial evaluated the efficacy and safety of TMZ in 70 elderly patients with GBM. ${ }^{15}$ The median overall survival was 25 weeks, and $26.5 \%$ of the patients had a complete or partial response to treatment, whereas $25 \%$ had stable disease. The KPS score improved in $32.9 \%$ of the patients, with 18 having achieved a KPS score of $\geq 70$. Global quality-of-life scores improved significantly over time, as did cognitive, social, physical, and role functioning scores. Motor dysfunction, drowsiness, and bladder control scores also improved before progression. To compare the various therapies, the Nordic Phase III trial randomly assigned 291 patients to TMZ treatment only $\left(200 \mathrm{mg} / \mathrm{m}^{2}\right.$ on a 5 -day schedule), hypofractionated RT (34 Gy in 3.4-Gy fractions), or standard RT (60 Gy in 2.0-Gy fractions). ${ }^{28}$ Temozolomide and hypofractionated RT were found to be superior to standard RT in those older than 70 years, although no significant effect was seen in those aged 60-70 years. Investigators found O6-methylguanine-DNA methyltransferase promoter methylation status to significantly affect the efficacy of TMZ, with patients who harbored methylated promoters having had improved survival over those who did not (9.7 vs 6.8 months, respectively; $\mathrm{p}=0.02$ ).

Because of the potential benefits of RT and TMZ in elderly patients with GBM, the utility of RT and concurrent TMZ has been investigated in this population. In a prospective study, Brandes et al. ${ }^{3}$ evaluated 79 patients who underwent postoperative RT, RT and adjuvant chemo-
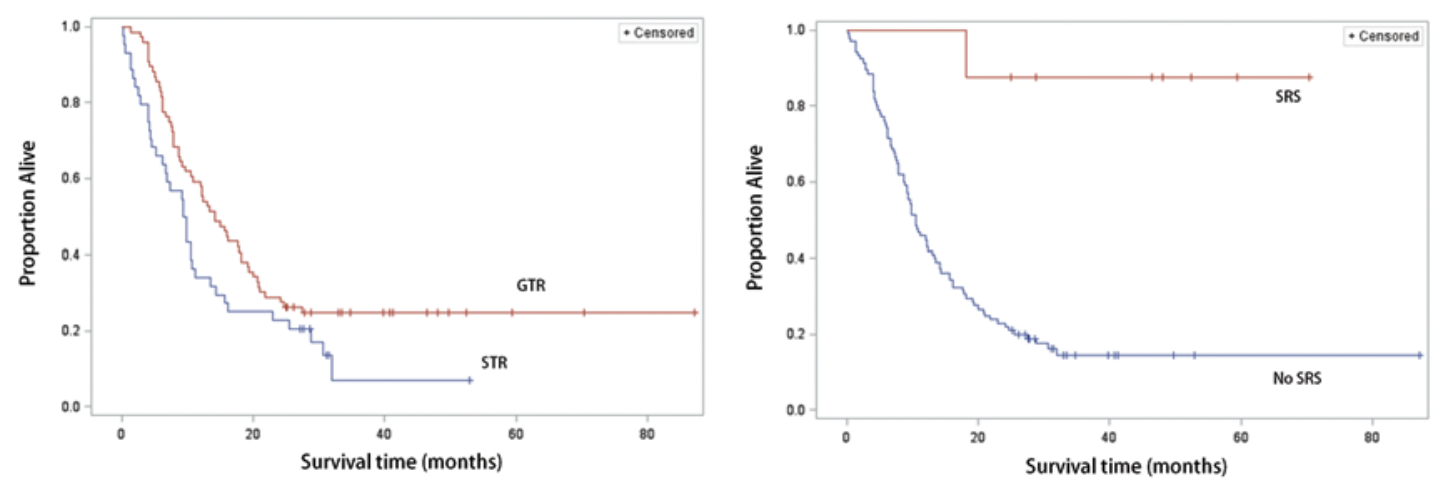

FIG. 3. Kaplan-Meier overall survival curves demonstrating significantly improved survival for patients who underwent GTR (left) and significantly improved survival for those who underwent SRS after tumor progression (right). Figure is available in color online only. 

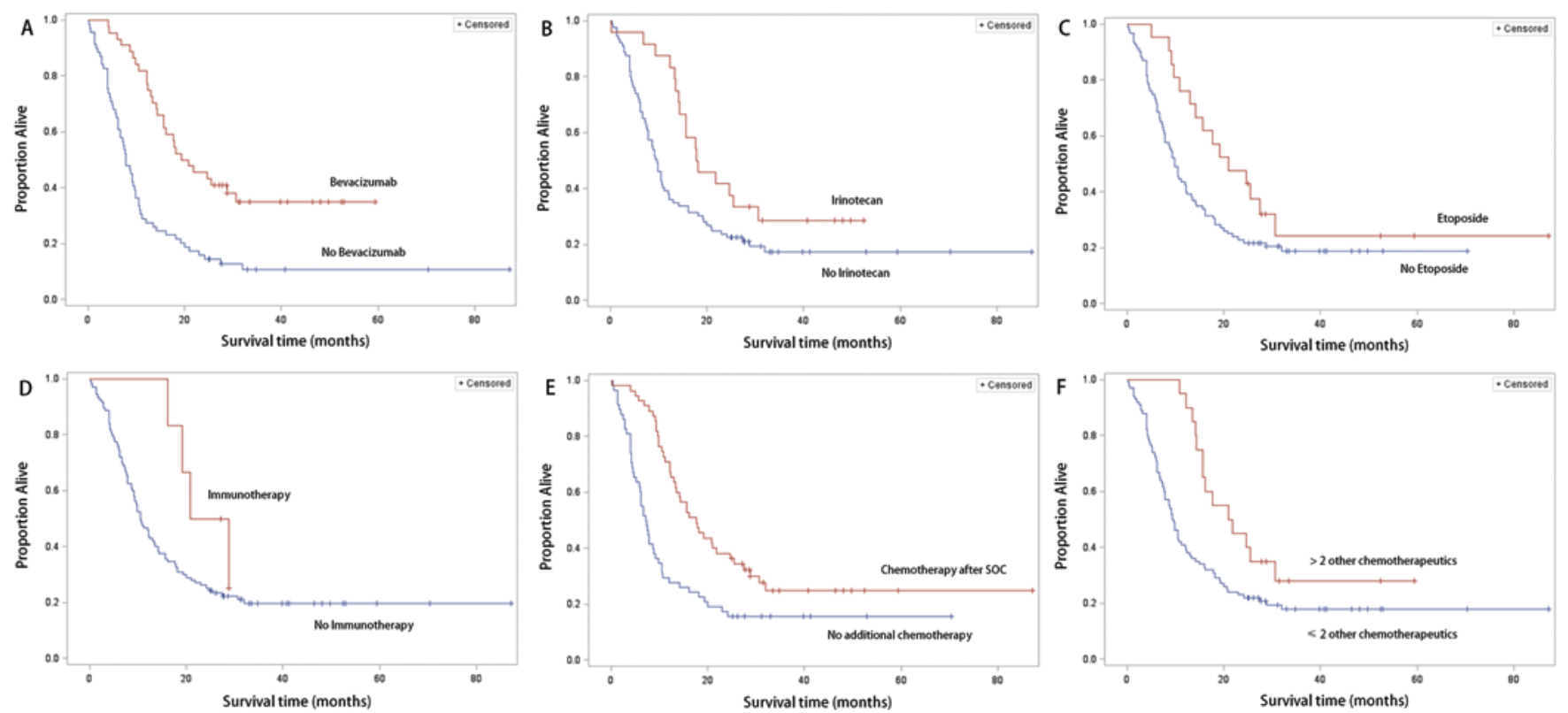

FIG. 4. Kaplan-Meier overall survival curves demonstrating significantly improved survival for patients who received bevacizumab (20.1 vs 7.9 months; $p<0.0001)(A)$, significantly improved survival for patients who received irinotecan (18.0 vs 9.7 months; $p=0.027)(B)$, and improved survival, which approached significance, for those who received etoposide (20.9 vs 9.9 months; $p=$ 0.052 ) (C). Immunotherapy did not have a significant effect on survival ( 24.8 vs 10.6 months; $p=0.17)$ (D), the use of chemotherapeutic agents following the standard of care (SOC) significantly improved survival (17.7 vs 7.2 months; $p=0.0004)(E)$, and the use of more than 2 chemotherapeutics after the standard of care significantly improved survival (21.3 vs 9.4 months; $p=0.018)(F)$. Figure is available in color online only.

therapy, and RT and adjuvant TMZ. Overall survival was found to be significantly higher in those who received adjuvant TMZ compared with those who received RT alone (14.9 vs 11.2 months, respectively; $\mathrm{p}=0.002$ ). In multivariate analysis, the effect of TMZ approached significance $(\mathrm{p}$ $=0.058$ ). Another study by Iwamoto et al..${ }^{20}$ who evaluated 394 elderly patients with GBM, demonstrated that, after adjusting for age, KPS score, EOR, and number of lesions, patients who received adjuvant chemotherapy had a 55\% decrease in the risk of death compared with those who received only RT. Similarly, another study that analyzed 105 elderly patients demonstrated that the addition of chemotherapy to RT resulted in significantly longer survival than RT alone (11.5 vs 4.5 months, respectively; $p=0.01$ ). ${ }^{46}$ Other institutional studies and those that used data linked with the Surveillance, Epidemiology, and End Results (SEER) and Medicare databases also reported that the addition of chemotherapy to RT improved outcomes. ${ }^{2,40}$ In our study, $97.3 \%$ of the patients received RT with concurrent TMZ, which prevents analysis of the effect of each individual therapy on outcomes. However, the prolonged survival reported in this study (12.0 months) is likely a result of this uniform aggressive treatment. Currently, the efficacy of RT with or without TMZ in elderly patients is being evaluated in an international Phase III trial conducted by the National Cancer Institute of Canada Clinical Trials Group (ClinicalTrials.gov registration number NCT00482677).

In light of advances in our understanding of GBM and the molecular alterations that frequently occur in these tumors, a variety of biologically based therapies have been developed. Bevacizumab, an antiangiogenic monoclonal antibody that binds to vascular endothelial growth factor (VEGF), received approval by the Food and Drug Administration in 2009 for recurrent GBM on the basis of several Phase II studies that demonstrated significant antitumor activity. 13,24,47,48 One large Phase II study that randomly assigned 167 patients to receive bevacizumab $(10 \mathrm{mg} / \mathrm{kg})$ alone or in combination with irinotecan reported 6-month progression-free survival rates of $42.6 \%$ and $50.3 \%$, and the median overall survival times were 9.2 and 8.7 months, respectively. ${ }^{13}$ It is interesting to note that the results of a recent study suggested that older patients experience a greater benefit from bevacizumab than younger patients..$^{30}$ This finding may be a result of older patients having a 1.4-foldhigher expression of VEGF-A. In this study, 38.9\% of the patients received bevacizumab. To our knowledge, ours is the first study to demonstrate that elderly patients experience significantly longer survival after treatment with bevacizumab than those who are not treated with beva-

TABLE 4. Stepwise multivariate analysis of various patient, tumor, and treatment characteristics that affected overall survival in elderly patients ( $\geq 65$ years old) with GBM

\begin{tabular}{lcl}
\hline \multicolumn{1}{c}{ Characteristic } & HR $(95 \% \mathrm{Cl})$ & $\mathrm{p} \mathrm{Value}$ \\
\hline Age & $1.06(1.02-1.10)$ & 0.0077 \\
\hline KPS score, preoperative & $0.97(0.95-0.99)$ & 0.0082 \\
\hline GTR & $0.70(0.46-1.08)$ & 0.10 \\
\hline Bevacizumab & $0.51(0.31-0.83)$ & 0.0067 \\
\hline
\end{tabular}


cizumab (20.1 vs 7.9 months, respectively; $p<0.0001)$. Bevacizumab was also an independent favorable prognostic factor after adjustment for age, KPS score, and EOR; it reduced the risk of death by nearly $50 \%$ (HR 0.51 [95\% CI $0.31-0.83] ; p=0.0067)$. Although this study demonstrated a significant survival benefit in elderly patients, current data do not support the use of bevacizumab in patients with newly diagnosed GBM. Two recent large Phase III trials failed to show an overall survival benefit, although an improvement in progression-free survival was observed. ${ }^{9,16}$ The efficacy of bevacizumab in combination with RT or TMZ is currently being evaluated in elderly patients with GBM in 2 randomized studies (ClinicalTrials.gov registration numbers NCT01443676 and NCT01149850).

Other agents, including irinotecan and etoposide, have been investigated for use in patients with recurrent GBM. Irinotecan is a topoisomerase I inhibitor that may induce apoptosis. ${ }^{11}$ This agent has demonstrated promise in the treatment of recurrent malignant glioma, especially in combination with TMZ or bevacizumab. ${ }^{49}$ In our univariate analysis, we have shown that irinotecan significantly improved survival in elderly patients over those who did not receive irinotecan (18.0 vs 9.7 months, respectively; $p=0.027$ ), although this effect was not significant in the multivariate analysis. Etoposide, a topoisomerase II inhibitor, has also been shown to have modest antitumor activity in malignant glioma. ${ }^{14,23,35}$ Our study also demonstrated that patients who received etoposide had improved outcomes over those who did not receive etoposide, although this result approached significance only in the univariate analysis (20.9 vs 9.9 months, respectively; $p=0.052)$. Although this study was not a randomized trial and we cannot conclusively recommend the use of any specific chemotherapeutics, we have shown more clearly the benefit of multiagent therapy after RT with concurrent TMZ. The administration of chemotherapy following the standard of care was associated with improved survival over those whose care did not follow the standard of care (17.7 vs 7.2 months, respectively; $p=0.0004)$. Furthermore, the use of more than 2 agents also led to increased survival over those who received 2 or fewer chemotherapeutics (21.3 vs 9.4 months, respectively; $p=0.018$ ). The investigation of multiagent regimens remains of great interest because of the often-complementary cytotoxic mechanisms of many drugs. In addition, various chemotherapeutics have been investigated in combination with bevacizumab because of its ability to alter tumor vasculature and thereby improve chemotherapy delivery. Such combinations have demonstrated improved outcomes in various cancers. ${ }^{19,39}$ For GBM, although some combination therapies have not demonstrated a significant effect on outcomes, others have shown promise in extending survival. ${ }^{7,45}$ Because of the promise of aggressive chemotherapeutic treatment in elderly patients with GBM, prospective studies using various multiagent regimens are needed not only to provide efficacy data but also to examine the effect on quality of life and cognitive functioning.

Currently, there are few data on the efficacy of SRS in elderly patients with recurrent GBM. Although studies have demonstrated that SRS and fractionated stereotactic RT provide a survival benefit in patients with tumor recur- rence, prospective trial data are lacking. ${ }^{36}$ In this study, we have demonstrated that the use of SRS in patients with focal tumor progression resulted in significantly longer survival than in those who did not receive radiosurgery (median not reached vs 10.5 months, respectively; $p=0.0006$ ). SRS was also significantly associated with increased survival in multivariate analysis (data not shown) when we controlled for only patient and tumor characteristics. However, the inclusion of chemotherapeutics in the model attenuated this significance. Because the benefit of SRS in this study may have been a result of unintended treatment selection bias, prospective studies are greatly needed to evaluate this effect.

This study had limitations, primarily because of its retrospective nature. As a result, it may be inherently biased and cannot provide causal data regarding the impact of treatments on survival. Also, this study was limited to patients treated at a single tertiary institution and therefore is affected by referral and selection biases, which are evident in the high percentage of tumors involving eloquent cortex. Furthermore, because all of the patients in this study underwent resection, this cohort of elderly patients may have been relatively healthy $(53.3 \%$ had a Charlson score of 0 ), thereby resulting in prolonged survival because of their ability to better tolerate the stresses of surgery and toxicities of chemotherapy. The high rate of GTR in this cohort of elderly patients may also support this notion, because nationwide studies have shown older patients to receive GTR significantly less frequently than younger patients. ${ }^{31}$ In addition, many patients in this study were enrolled in a variety of clinical trials, such as those evaluating combination therapies and immunotherapy. As a result, the varying treatment and follow-up protocols may have affected patient survival. Furthermore, patient enrollment in clinical trials may itself affect outcomes; studies have demonstrated improved survival of patients who participate in clinical trials irrespective of the arm. ${ }^{41}$ Nonetheless, we evaluated a variety of chemotherapeutics and for the first time demonstrated that bevacizumab provides a significant survival benefit for these patients.

\section{Conclusions}

With this study, we demonstrated that GTR conferred on elderly patients with GBM a modest survival benefit, which suggests that safe maximal resection is warranted. In addition, we report that bevacizumab significantly increased the overall survival of elderly patients with GBM, and older age and preoperative KPS score were also significant prognostic factors according to our multivariate analysis. Although elderly patients with GBM have a poor prognosis, they may experience enhanced survival after administration of the standard of care and additional chemotherapeutics after progression. The results of randomized trials currently in progress are eagerly awaited to provide more definitive data on the appropriate management of elderly patients with GBM.

\section{Acknowledgments}

This study was in part funded by a grant from the American Federation for Aging Research provided to Dr. Babu. The fund- 
ing organization did not have any role in the design and conduct of the study; the collection, management, analysis, and interpretation of the data; and preparation, review, or approval of the manuscript. During this study, Dr. Adamson also served as Chief of Neurosurgery, Department of Veterans Affairs Medical Center, Durham, North Carolina. The contents of this manuscript do not represent the views of the Department of Veterans Affairs or the United States Government

\section{References}

1. Adamson C, Kanu OO, Mehta AI, Di C, Lin N, Mattox AK, et al: Glioblastoma multiforme: a review of where we have been and where we are going. Expert Opin Investig Drugs 18:1061-1083, 2009

2. Barnholtz-Sloan JS, Williams VL, Maldonado JL, Shahani D, Stockwell HG, Chamberlain M, et al: Patterns of care and outcomes among elderly individuals with primary malignant astrocy toma. J Neurosurg 108:642-648, 2008

3. Brandes AA, Vastola F, Basso U, Berti F, Pinna G, Rotilio A, et al: A prospective study on glioblastoma in the elderly. Cancer 97:657-662, 2003

4. Chaichana KL, Chaichana KK, Olivi A, Weingart JD, Bennett R, Brem H, et al: Surgical outcomes for older patients with glioblastoma multiforme: preoperative factors associated with decreased survival. Clinical article. J Neurosurg 114:587-594, 2011

5. Chakrabarti I, Cockburn M, Cozen W, Wang YP, PrestonMartin S: A population-based description of glioblastoma multiforme in Los Angeles County, 1974-1999. Cancer 104:2798-2806, 2005

6. Charlson ME, Pompei P, Ales KL, MacKenzie CR: A new method of classifying prognostic comorbidity in longitudinal studies: development and validation. J Chronic Dis 40:373383, 1987

7. Chen C, Ravelo A, Yu E, Dhanda R, Schnadig I: Clinical outcomes with bevacizumab-containing and non-bevacizumabcontaining regimens in patients with recurrent glioblastoma from US community practices. J Neurooncol 122:595-605, 2015

8. Chinot OL, Barrie M, Frauger E, Dufour H, Figarella-Branger D, Palmari J, et al: Phase II study of temozolomide without radiotherapy in newly diagnosed glioblastoma multiforme in an elderly populations. Cancer 100:2208-2214, 2004

9. Chinot OL, Wick W, Mason W, Henriksson R, Saran F, Nishikawa R, et al: Bevacizumab plus radiotherapy-temozolomide for newly diagnosed glioblastoma. N Engl J Med 370:709-722, 2014

10. Combs SE, Wagner J, Bischof M, Welzel T, Wagner F, Debus J, et al: Postoperative treatment of primary glioblastoma multiforme with radiation and concomitant temozolomide in elderly patients. Int J Radiat Oncol Biol Phys 70:987-992, 2008

11. Creemers GJ, Lund B, Verweij J: Topoisomerase I inhibitors: topotecan and irenotecan. Cancer Treat Rev 20:73-96, 1994

12. Fiorica F, Berretta M, Colosimo C, Stefanelli A, Ursino S, Zanet E, et al: Glioblastoma in elderly patients: safety and efficacy of adjuvant radiotherapy with concomitant temozolomide. Arch Gerontol Geriatr 51:31-35, 2010

13. Friedman HS, Prados MD, Wen PY, Mikkelsen T, Schiff D, Abrey LE, et al: Bevacizumab alone and in combination with irinotecan in recurrent glioblastoma. J Clin Oncol 27:47334740, 2009

14. Fulton D, Urtasun R, Forsyth P: Phase II study of prolonged oral therapy with etoposide (VP16) for patients with recurrent malignant glioma. J Neurooncol 27:149-155, 1996

15. Gállego Pérez-Larraya J, Ducray F, Chinot O, Catry-Thomas I, Taillandier L, Guillamo JS, et al: Temozolomide in elderly patients with newly diagnosed glioblastoma and poor performance status: an ANOCEF phase II trial. J Clin Oncol 29:3050-3055, 2011

16. Gilbert MR, Dignam JJ, Armstrong TS, Wefel JS, Blumenthal DT, Vogelbaum MA, et al: A randomized trial of bevacizumab for newly diagnosed glioblastoma. N Engl J Med 370:699-708, 2014

17. Glantz M, Chamberlain M, Liu Q, Litofsky NS, Recht LD: Temozolomide as an alternative to irradiation for elderly patients with newly diagnosed malignant gliomas. Cancer 97:2262-2266, 2003

18. Hoffman S, Propp JM, McCarthy BJ: Temporal trends in incidence of primary brain tumors in the United States, 1985-1999. Neuro Oncol 8:27-37, 2006

19. Hurwitz H, Fehrenbacher L, Novotny W, Cartwright T, Hainsworth J, Heim W, et al: Bevacizumab plus irinotecan, fluorouracil, and leucovorin for metastatic colorectal cancer. N Engl J Med 350:2335-2342, 2004

20. Iwamoto FM, Cooper AR, Reiner AS, Nayak L, Abrey LE: Glioblastoma in the elderly: the Memorial Sloan-Kettering Cancer Center Experience (1997-2007). Cancer 115:37583766, 2009

21. Iwamoto FM, Reiner AS, Panageas KS, Elkin EB, Abrey LE: Patterns of care in elderly glioblastoma patients. Ann Neurol 64:628-634, 2008

22. Keime-Guibert F, Chinot O, Taillandier L, Cartalat-Carel S, Frenay M, Kantor G, et al: Radiotherapy for glioblastoma in the elderly. N Engl J Med 356:1527-1535, 2007

23. Kesari S, Schiff D, Doherty L, Gigas DC, Batchelor TT, Muzikansky A, et al: Phase II study of metronomic chemotherapy for recurrent malignant gliomas in adults. Neuro Oncol 9:354-363, 2007

24. Kreisl TN, Kim L, Moore K, Duic P, Royce C, Stroud I, et al: Phase II trial of single-agent bevacizumab followed by bevacizumab plus irinotecan at tumor progression in recurrent glioblastoma. J Clin Oncol 27:740-745, 2009

25. Kuhnt D, Becker A, Ganslandt O, Bauer M, Buchfelder M, Nimsky C: Correlation of the extent of tumor volume resection and patient survival in surgery of glioblastoma multiforme with high-field intraoperative MRI guidance. Neuro Oncol 13:1339-1348, 2011

26. Lacroix M, Abi-Said D, Fourney DR, Gokaslan ZL, Shi W, DeMonte F, et al: A multivariate analysis of 416 patients with glioblastoma multiforme: prognosis, extent of resection, and survival. J Neurosurg 95:190-198, 2001

27. Lamborn KR, Chang SM, Prados MD: Prognostic factors for survival of patients with glioblastoma: recursive partitioning analysis. Neuro Oncol 6:227-235, 2004

28. Malmström A, Grønberg BH, Marosi C, Stupp R, Frappaz D, Schultz H, et al: Temozolomide versus standard 6-week radiotherapy versus hypofractionated radiotherapy in patients older than 60 years with glioblastoma: the Nordic randomised, phase 3 trial. Lancet Oncol 13:916-926, 2012

29. Minniti G, De Sanctis V, Muni R, Filippone F, Bozzao A, Valeriani M, et al: Radiotherapy plus concomitant and adjuvant temozolomide for glioblastoma in elderly patients. J Neurooncol 88:97-103, 2008

30. Nghiemphu PL, Liu W, Lee Y, Than T, Graham C, Lai A, et al: Bevacizumab and chemotherapy for recurrent glioblastoma: a single-institution experience. Neurology 72:1217-1222, 2009

31. Noorbakhsh A, Tang JA, Marcus LP, McCutcheon B, Gonda $\mathrm{DD}$, Schallhorn CS, et al: Gross-total resection outcomes in an elderly population with glioblastoma: a SEER-based analysis. J Neurosurg 120:31-39, 2014

32. Olson JJ, Ryken T: Guidelines for the treatment of newly diagnosed glioblastoma: introduction. J Neurooncol 89:255258, 2008

33. Orringer D, Lau D, Khatri S, Zamora-Berridi GJ, Zhang K, 
Wu C, et al: Extent of resection in patients with glioblastoma: limiting factors, perception of resectability, and effect on survival. J Neurosurg 117:851-859, 2012

34. Oszvald A, Güresir E, Setzer M, Vatter H, Senft C, Seifert V, et al: Glioblastoma therapy in the elderly and the importance of the extent of resection regardless of age. J Neurosurg 116:357-364, 2012

35. Reardon DA, Desjardins A, Vredenburgh JJ, Gururangan S, Sampson JH, Sathornsumetee S, et al: Metronomic chemotherapy with daily, oral etoposide plus bevacizumab for recurrent malignant glioma: a phase II study. Br J Cancer 101:1986-1994, 2009

36. Romanelli P, Conti A, Pontoriero A, Ricciardi GK, Tomasello F, De Renzis C, et al: Role of stereotactic radiosurgery and fractionated stereotactic radiotherapy for the treatment of recurrent glioblastoma multiforme. Neurosurg Focus 27(6):E8, 2009

37. Sanai N, Berger MS: Glioma extent of resection and its impact on patient outcome. Neurosurgery 62:753-764, 264266, 2008

38. Sanai N, Polley MY, McDermott MW, Parsa AT, Berger MS: An extent of resection threshold for newly diagnosed glioblastomas. J Neurosurg 115:3-8, 2011

39. Sandler A, Gray R, Perry MC, Brahmer J, Schiller JH, Dowlati A, et al: Paclitaxel-carboplatin alone or with bevacizumab for non-small-cell lung cancer. N Engl J Med 355:2542-2550, 2006

40. Scott JG, Suh JH, Elson P, Barnett GH, Vogelbaum MA, Peereboom DM, et al: Aggressive treatment is appropriate for glioblastoma multiforme patients 70 years old or older: a retrospective review of 206 cases. Neuro Oncol 13:428-436, 2011

41. Shahar T, Nossek E, Steinberg DM, Rozovski U, Blumenthal DT, Bokstein F, et al: The impact of enrollment in clinical trials on survival of patients with glioblastoma. J Clin Neurosci 19:1530-1534, 2012

42. Stark AM, Nabavi A, Mehdorn HM, Blömer U: Glioblastoma multiforme-report of 267 cases treated at a single institution. Surg Neurol 63:162-169, 2005

43. Stummer W, Reulen HJ, Meinel T, Pichlmeier U, Schumacher W, Tonn JC, et al: Extent of resection and survival in glioblastoma multiforme: identification of and adjustment for bias. Neurosurgery 62:564-576, 2008

44. Stupp R, Mason WP, van den Bent MJ, Weller M, Fisher B, Taphoorn MJ, et al: Radiotherapy plus concomitant and adjuvant temozolomide for glioblastoma. N Engl J Med 352:987-996, 2005

45. Taal W, Oosterkamp HM, Walenkamp AM, Dubbink HJ,
Beerepoot LV, Hanse MC, et al: Single-agent bevacizumab or lomustine versus a combination of bevacizumab plus lomustine in patients with recurrent glioblastoma (BELOB trial): a randomised controlled phase 2 trial. Lancet Oncol 15:943-953, 2014

46. Tanaka S, Meyer FB, Buckner JC, Uhm JH, Yan ES, Parney IF: Presentation, management, and outcome of newly diagnosed glioblastoma in elderly patients. J Neurosurg 118:786-798, 2012

47. Vredenburgh JJ, Desjardins A, Herndon JE II, Dowell JM, Reardon DA, Quinn JA, et al: Phase II trial of bevacizumab and irinotecan in recurrent malignant glioma. Clin Cancer Res 13:1253-1259, 2007

48. Vredenburgh JJ, Desjardins A, Herndon JE II, Marcello J, Reardon DA, Quinn JA, et al: Bevacizumab plus irinotecan in recurrent glioblastoma multiforme. J Clin Oncol 25:4722-4729, 2007

49. Vredenburgh JJ, Desjardins A, Reardon DA, Friedman HS: Experience with irinotecan for the treatment of malignant glioma. Neuro Oncol 11:80-91, 2009

50. Wrensch M, Minn Y, Chew T, Bondy M, Berger MS: Epidemiology of primary brain tumors: current concepts and review of the literature. Neuro Oncol 4:278-299, 2002

\section{Disclosure}

The authors report no conflict of interest concerning the materials or methods used in this study or the findings specified in this paper.

\section{Author Contributions}

Conception and design: Adamson, Babu. Acquisition of data: Babu, Komisarow, Agarwal, Rahimpour, Iyer, Britt, Karikari. Analysis and interpretation of data: Adamson, Babu, Thomas. Drafting the article: Babu, Iyer. Critically revising the article: Adamson, Babu, Komisarow, Agarwal, Rahimpour, Karikari, Grossi, Thomas, Friedman. Reviewed submitted version of manuscript: Adamson, Babu, Komisarow, Agarwal, Rahimpour, Britt, Karikari, Grossi, Thomas, Friedman. Statistical analysis: Babu, Thomas. Study supervision: Adamson, Friedman.

\section{Correspondence}

Cory Adamson, Department of Neurosurgery, Emory University Hospital, 1364 Clifton Road NE, Atlanta, GA 30322. email: cory. adamson@emory.edu. 